\title{
Insecticide Resistant Anopheles Gambiae Have Enhanced Longevity but Reduced biting Frequency and Reproductive Fitness
}

Joyce K. Osoro

Masinde Muliro University of Science and Technology

Maxwell G. Machani

Kenya Medical Research Institute

Eric Ochomo

Kenya Medical Research Institute

Christine Wanjala

Masinde Muliro University of Science and Technology

\section{Elizabeth Omukunda}

Masinde Muliro University of Science and Technology

Andrew K. Githeko

Kenya Medical Research Institute

\section{Guiyun Yan}

University of California

Yaw A. Afrane ( $\square$ yaw_afrane@yahoo.com )

University of Ghana Medical School, University of Ghana

\section{Research Article}

Keywords: Anopheles gambiae, insecticide resistance, gonotrophic cycle, fecundity, reproductive fitness, longevity

Posted Date: October 25th, 2021

DOl: https://doi.org/10.21203/rs.3.rs-998001/v1

License: (c) (i) This work is licensed under a Creative Commons Attribution 4.0 International License. Read Full License 


\section{Abstract}

Background: Widespread insecticide resistance in African malaria vectors raises concerns over the potential to compromise malaria vector control interventions. Understanding the evolution of resistance mechanisms, and whether the selective disadvantages are large enough to be useful in resistance management or designing suitable control strategies is crucial. This study assessed whether insecticide resistance to pyrethroids has an effect on the gonotrophic cycle and reproductive potential of malaria vector Anopheles gambiae.

Methods: Comparative tests were performed with a pyrethroid resistant and susceptible colonies of Anopheles gambiae colonized from the same geographical area, and the reference Kisumu strain was used as a control. Adult females aged 2 days old were given a blood meal and kept separately for individual egg laying. The number of days taken to lay eggs post blood feeding was recorded to determine the length of gonotrophic cycle. To measure adult longevity and reproduction potential, newly emerged males and females of equal numbers were aspirated into a cage and females allowed to blood feed daily. The number of eggs laid and the surviving mosquitoes were recorded daily to determine fecundity, net reproduction rate, intrinsic growth rate and adult longevity.

Results: Overall, the resistant females had a significantly longer (1.8 days) gonotrophic cycle than susceptible females $\left(F_{2,13}=9.836, P<0.01\right)$. The proportion of resistant females that laid eggs was lower $31.30 \%(94 / 300)$ compared to $54 \%(162 / 300)$ in the susceptible colony and $65.7 \%(197 / 300)$ in the Kisumu strain. The mean number of eggs laid per female was significantly lower in the resistant colony $(88.02 \pm 20)$ compared to the susceptible colony $(104.9 \pm .28 .8)$ and the Kisumu strain $(97.6 \pm 34.8)$. The adult longevity was significantly higher for resistant (39.7 \pm 1.6 days) compared to susceptible $(29.9 \pm$ 1.7 days $)$ and the Kisumu strain was $(29.6 \pm 1.1$ days $)\left(F_{2,8}=45.05, P<0.0001\right)$. Resistant colony exhibited a lower fecundity (4.3 eggs/females/day) and net reproductive rate (2.6 offsprings/female/generation) compared to the susceptible colony (8.6 eggs/female/day; 4.7 off springs/female/generation respectively) and Kisumu strain (9.7 eggs/female/day; 4.1 off springs/female/generation respectively).

Conclusion: The study suggests high fitness cost on reproductive parameters of pyrethroid resistant mosquitoes particularly on the duration of gonotrophic cycle, fecundity and net reproductive rate. These fitness costs are likely associated with maintaining both target site and metabolic mechanisms of resistance to pyrethroids. Despite these costs, the resistant mosquitoes had a longer longevity. The results of this study are critical to determining the extent to which insecticide resistance interacts with mosquito reproduction potential and should provide key information in the design of more targeted insecticide resistance management strategies.

\section{Background}


Current efforts to control malaria rely heavily on insecticide-based interventions such as large-scale distribution of long-lasting insecticidal nets (LLINs) and indoor residual spraying (IRS) ${ }^{1}$. Pressure placed upon mosquitoes by the rapid scaling-up of vector control interventions and the use of similar chemicals for agricultural activities have been reported to select for phenotypes with increased ability to survive the insecticides used, gradually impacting vector control efforts ${ }^{2}$. A number of studies have demonstrated that the major insecticide resistance mechanisms involved include decreased sensitivity of the target proteins and increasing activity of detoxifying enzymes (metabolic resistance) ${ }^{3}$. Although these mechanisms confer a significant advantage to the bearers in the presence of insecticides, they have also been associated with a series of side effects in the life history traits of the insect population ${ }^{4}$, which include their biting rate, fecundity and survivorship. However, the expression of these side effects associated with insecticide resistance in Anopheles gambiae is unclear.

The biting rate of a vector is dependent on the gonotrophic cycle, which is defined as the time interval between blood feeding and oviposition ${ }^{5,6}$ and is dependent on the search for a blood meal, blood-feeding and digestion, egg maturation and the availability of a suitable oviposition site ${ }^{6}$. This, therefore, dictates the biting frequency of a vector impacting vectorial capacity and transmission dynamics of malaria ${ }^{6,7}$. Survivorship and fecundity are important determinants of the population growth dynamics of mosquitoes ${ }^{8,9}$. Adult survivorship is one of the fundamental factors of vectorial capacity, a change due to fitness cost could have profound effect on the disease transmission risks and epidemiology of malaria ${ }^{8}$. For instance, a mosquito needs to survive beyond the extrinsic incubation period of the Plasmodium parasites to be able to transmit malaria and a longer lifespan guarantees a potential of biting many hosts 7,9 . Fecundity, a trait measured by the number of offspring an individual mosquito can produce, is a major fitness component since it determines the reproductive rate and intrinsic growth rate of a population 5,10 . Reproductive rate is the average number of offspring produced by a female individual in her lifetime, whilst, intrinsic growth rate is defined as the number of progeny born to each female mosquito per unit time ${ }^{5}$. Reproductive rate and intrinsic growth rate impact directly on the population of mosquitoes which is a major component of vectorial capacity of malaria transmission.

Studies have proven that insecticide resistance impacts the physiological processes of mosquito vectors. These biological processes include adult survivorship, fecundity, blood-feeding, male mating success, development of immature stages and also vector competence ${ }^{11-16}$. Most of these studies were generally carried out under optimal laboratory conditions and involved unrelated resistant and susceptible strains which often differ in many other genes as they originate differently. In addition, the use of reference susceptible strains singly, may not give a true comparison of fitness costs as this population has been maintained in the laboratory for decades and therefore may adapt to laboratory conditions. This study explored the fitness costs of insecticide resistance on the gonotrophic cycle, fecundity and adult survivorship under a semi-natural setting using established colonies of pyrethroid-resistant and susceptible An. gambiae with the same origin ${ }^{17}$. 


\section{Materials And Methods}

\section{Mosquito population used in the study}

The population of mosquitoes used in this study consisted of a resistant colony of An. gambiae selected through exposure to deltamethrin insecticide (hereafter referred as a resistant colony) and unselected susceptible colony of An. gambiae that was raised in the absence of insecticides over several generations (hereafter referred as a susceptible colony). Both resistant and susceptible colonies originated from the same mother colony collected from Bungoma, western Kenya ${ }^{17}$.

\section{Resistant colony}

The colony was resistant to deltamethrin with mortality of $20 \%$. The colony showed both metabolic resistance, through increased monooxygenases enzyme activity, and Kdr mutations (V1014S and V1014F $)^{17}$. The 6 th generation was used for this study.

\section{Susceptible colony}

This colony did not undergo selection at any generation but was monitored for resistance after every generation. This colony had lost phenotypic resistance to deltamethrin after 13 generations (Mortality rate $97.8 \%$ ). Only Kdr mutation (L1014S) was detected as it was already fixed in the parent population ${ }^{17}$. The 13th generation was used for this study. The generation difference between the resistant and susceptible colony was due to the delayed development in the selected resistant colony.

\section{Kisumu reference laboratory strain}

The Kisumu reference laboratory strain which has been colonized since $1954^{18}$ and is free of any detectable insecticide resistance mechanism was used as a control susceptible strain in all bioassays.

These An. gambiae s.s mosquitoes that were used in this study were reared in the insectary at KEMRI/CGHR under standard conditions $\left(25 \pm 2^{\circ} \mathrm{C} ; 80 \% \pm 4 \%\right.$ relative humidity with a $12 \mathrm{~h}: 12 \mathrm{~h}$ light/dark cycle). Larvae were fed on tetramin baby fish food and brewer's yeast daily and adults were maintained on $10 \%$ sugar solution. The resistant and the susceptible colonies used were reared in three lineages as replicates.

\section{Experimental design}




\section{Determination of the gonotrophic cycle of the experimental mosquitoes}

Newly emerged (one day old) males and females from the resistant, susceptible and the Kisumu reference strain were put in separate cages to allow time for mating. On the second day, the females were given a blood meal for 20 minutes. After 24 hours ( 1 day) post-feeding, three hundred (300) fully blood-fed females from each of the colonies were then transferred to individual oviposition cups that were lined with filter papers on moist cotton wool. The eggs laid by each mosquito were counted and recorded and the number of days taken to lay eggs after blood-feeding was also recorded in order to determine the length of the gonotrophic cycle. The number of mosquitoes that laid eggs and the number of eggs laid were recorded to determine the daily egg-laying rate and the size of egg batches.

\section{Adult Longevity and fecundity of resistant and susceptible An. gambiae}

One hundred (100) newly emerged (one day old) females and males from each of the resistant and susceptible colonies and the Kisumu reference strain were placed separately in a $30 \times 30 \times 30 \mathrm{~cm}$ metalframed cages covered with nylon netting. Cages were suspended from the roof, $2 \mathrm{~m}$ above the ground in the huts of the MalariaSphere at the Centre for Global Health Research of the Kenya Medical Research Institute in Kisumu, Kenya. Grease was smeared on the suspension twine to prevent ants from interfering with the experiment. Mosquitoes in each cage were fed on $10 \%$ sucrose solution through a cotton ball. Females in each cage were given a blood meal daily for 20 minutes. An oviposition substrate consisting of a petri dish lined with a filter paper on wet cotton wool was provided for oviposition. The number of

eggs laid were picked and counted under a dissecting microscope daily to determine fecundity ${ }^{19} 20$. Dead male and female mosquitoes were recorded and removed from the cage daily. The experiment was done in three replicates for each of the resistant, susceptible and Kisumu strains.

\section{Ethical statement}

Ethical approval was obtained from the Kenya Medical Research Institute Scientific and Ethical Review Unit (SERU) under approval number SSC 3434.

\section{Data analysis}

Gonotrophic cycle length was calculated as the average number of days taken for a mosquito to oviposit eggs after taking a blood meal for each colony. The proportion of mosquitoes that laid eggs was calculated as the number of mosquitoes that were able to lay eggs per colony. The average age-specific survivorship for the females was calculated for each colony as the average number of days that the 
mosquitoes lived per colony. Fecundity was calculated as the average number of eggs laid per individual mosquito per day. The net reproductive rate $\left(R_{0}\right)$ for each of the three colonies was calculated based on the daily survivorship and fecundity schedule. $R_{0}$ is defined as the average number of offspring a female individual in a population will produce in her lifetime and is calculated as $R 0=£\left(I_{\mathrm{x}} m_{\mathrm{x}}\right)$, where $I_{\mathrm{x}}$ is the age-specific survivorship, and $m_{\mathrm{x}}$ is the age-specific fecundity per mosquito. Intrinsic per-capita growth Rate $(r)$, defined as the number of progeny born to each female mosquito per unit of time, was calculated as $r=\operatorname{Ln}\left(R_{0}\right) / G$ where $G=£ l_{x} m_{x} x / £ l_{x} m_{x}$ and $x$ is mosquito age ${ }^{5,19}$. Analysis of variance (ANOVA) was used to determine the effect of insecticide resistance on the gonotrophic cycle, fecundity, net reproductive rate and per-capita intrinsic growth rate of the resistant, susceptible and Kisumu strain mosquitoes. Tukey (HSD) was used to test the significance of difference in the reproductive parameters among the resistant, susceptible and Kisumu strain An. gambiae

\section{Results}

\section{Duration of gonotrophic cycle}

The average duration of gonotrophic cycle measured from the time of blood-feeding to egg-laying for the resistant females was longer ( $6.1 \pm 0.8$ days) than the susceptible females ( $4.3 \pm 1.1$ days) and $3.7 \pm 0.2$ days for the Kisumu strain $\left(F_{2,13}=9.836, P<0.003\right)$. The average duration of the gonotrophic cycle for the resistant females was significantly longer by 1.8 days than the susceptible colony $\left(F_{2,13}=9\right.$. 836, $P$ $<0.01$; Table 2). Although the duration of the gonotrophic cycle for susceptible females was higher compared to the Kisumu strain, this was not statistically significant $\left(F_{2,13}=9.836, P=0.570\right)$. The proportion of resistant females that successfully laid eggs was lower $31.30 \%(94 / 300)$ compared to $54 \%$ $(162 / 300)$ susceptible colony and $65.7 \%(197 / 300)$ Kisumu strain. The number of resistant females that laid eggs was significantly lower than the susceptible females $\left(F_{2,11}=0.461, P<0.05\right)$. The average number of eggs laid by an individual mosquito in the resistant colony was lower (88.02 \pm 20$)$ compared to the susceptible colony $(104.9 \pm .28 .8)$ and the Kisumu strain $(97.6 \pm 34.8)$. The fecundity of the resistant colony was significantly lower than the susceptible colony by 16 eggs per mosquito $\left(F_{2,449}=\right.$ $6.786, \mathrm{P}<0.001)$. Although the number of eggs laid per individual mosquito in the Kisumu colony was higher than the resistant colony, this was not statistically significant $(P=0.64)$.

\section{Survivorship and fecundity of resistant and susceptible Anopheles gambiae.}

The average longevity for the resistant colony was $39.7 \pm 1.6$ days while the susceptible colony was 29.9 \pm 1.7 days and the Kisumu strain was $29.6 \pm 1.1$ days $\left(F_{2,8}=45.05, P<0.0001\right.$; Table 2$)$. The average longevity recorded by the resistant colony was significantly longer (by 10 days) than the susceptible colony and Kisumu strain mosquitoes $\left(F_{2,8}=45.05, P<0.05\right)$. Resistant females survived slightly longer (85 days), with a median longevity of 43 days than the susceptible colony that lived for 67 days with a 
median longevity of 34 days and Kisumu strain that survived for 65 days having a median longevity of 33 days (Figure 1 ).

The average fecundity of the 3 replicates of resistant females placed in different cages was lower (4.3 eggs per female per day) than susceptible females (8.6 eggs per females per day) and Kisumu strain (9.7 eggs per female per day) $\left(F_{2,9}=38.16, P<0.0004\right)$. The average fecundity for resistant females was significantly lower by 4.3 eggs per day than the susceptible females $\left(F_{2,9}=38.16, P<0.001\right.$; Table 2$)$. The fecundity was slightly higher in Kisumu reference strain than the susceptible colony, but the difference was not statistically significant $\left(F_{2,9}=38.16, P>0.05\right)$.

\section{Reproductive fitness of resistant and susceptible mosquitoes}

The net reproductive rate calculated for resistant females was lower (2.6 off spring/female/generation) compared to susceptible females (4.7 off spring/female/generation) and Kisumu strain (4.1

offspring/female/generation, Table 2). Susceptible females exhibited a 2-fold increase in net reproductive rate over the resistant females $\left(F_{2,9}=20.24, P<0.002\right)$, indicating low reproduction for mosquitoes expressing physiological resistance. There was no significant difference in net reproductive rate between the susceptible colony and Kisumu reference strain $\left(F_{2,9}=20.24, P>0.05\right)$.

Similarly, resistant females exhibited a lower intrinsic growth rate ( 0.215 females /day) compared to susceptible females $(0.285)$ and Kisumu strain (0.241). The intrinsic growth rate was significantly higher for susceptible females compared to resistant females $\left(F_{2,9}=41.98, P<0.0002\right.$; Table 2$)$ suggesting that susceptible females will have a major competitive advantage over mosquitoes carrying resistant genes.

\section{Discussion}

The emergence and spread of insecticide resistance in malaria vectors of sub-Saharan Africa raise concerns over the control of the disease ${ }^{21}$. Despite the observed resistance, little is known about how insecticide resistance influences the biting frequency, survivorship and reproductive fitness of malaria vectors. Therefore, understanding the effects and mechanisms of insecticide resistance on vector adaptation capacities is crucial to the conservation of susceptibility and development of more effective resistance management strategies. In this study, we evaluated reproductive fitness of two progenies of An. gambiae with same origin but different insecticide resistance profiles when exposed to pyrethroid insecticides. Overall, results showed that the resistant mosquitoes have a fitness disadvantage on reproductive parameters in comparison to the susceptible strain, suggesting the possible accumulation of deleterious effects of insecticide resistance. However the survivorship of the resistant mosquitoes was significantly longer compared to the susceptible mosquitoes. 
The determination of the gonotrophic cycle, which is a measure of biting frequency is an important parameter in estimating the opportunities for acquisition and transmission of parasites ${ }^{22}$. This study observed a longer gonotrophic cycle in resistant females compared to their susceptible counterparts suggesting a possible lower human biting rate which could result in lower malaria transmission. This findings corroborate with Mebrahtu, et al. ${ }^{23}$ who observed a delay in the duration of laying eggs for resistant Aedes aegypti compared to the susceptible females. The relatively longer gonotrophic period in the resistant females points towards a lower blood digestion rate that may result from the altered physiology of the female mosquito. Although, this study did not measure the blood meal size in the resistant and susceptible mosquiotes, some studies have observed a significant reduction of the relative amount of ingested blood in resistant mosquitoes compared to susceptible counterparts ${ }^{16,23-25}$. The longer gonotrophic cycle observed in the resistant mosquitoes may suggest reduced biting frequencies and potentially to less transmission of malaria parasites in nature, when compared to the susceptible counterparts. However, such mosquitoes with elongated gonotrophic cycle may have multiple blood meals before laying eggs, which could increase the chances of picking the malaria parasite and having high potential for transmitting the disease.

The resistant mosquitoes were observed to live longer than susceptible females suggesting that these females could have a longer infective lifespan which may have implications for malaria transmission. The increase in longevity observed in the resistant colony could be a result of the extended larval stage ${ }^{26}$, allowing for a greater accumulation of nutritional resources partially compensating for the losses associated with maintaining the resistance mechanism ${ }^{27}$. Studies with the same colony of mosquitoes showed an extended larval development time of the resistant mosquitoes compared to the susceptible ones $^{26}$. This trait may be critical in promoting the maintenance of resistant individuals in the field, thereby hindering the effectiveness of insecticide resistance management actions. This finding agrees with previous reports of resistant An. funestus ${ }^{14}$ that had resistant alleles living longer compared to those with susceptible alleles. On the contrary, there are studies that reported shorter adult lifespan in resistant females such as pyrethroid resistant An. coluzzii ${ }^{16}$ and Aedes aegypti with kdr mutations ${ }^{24}$ which were associated with decreased longevity than their susceptible counterparts. In the field scenario, longer survival of resistant mosquitoes will favour the completion of sporogonic cycle of malaria parasites, increased biting of people and potentially transmit disease more than susceptible mosquitoes.

Reduction in the number of egg-laying females was observed in the resistant colony. The number of eggs laid per individual resistant female was lower than in the susceptible colony implying reduced fecundity and egg-laying ability. The variation in fecundity observed may suggests that the nutrients obtained during the blood meal were used for maintaining other processes linked to the survival of resistant females instead of egg production. These findings corroborate with Mebrahtu, et al. ${ }^{23}$ and Sy, et al. ${ }^{25}$ who observed a reduction in the number of eggs laid by insecticide-resistant strains of Ae. aegypti and An coluzzii respectively. The reduced ability of resistant mosquitoes to lay eggs could also be attributed to lower insemination rates in insecticide-resistant mosquitoes as reported in other studies ${ }^{23}$, although this trait was not assessed in this study. The long gonotrophic cycle would also make the resistant 
mosquitoes to take blood meals less often which reduces the egg-laying frequency hence explaining the reduced fecundity of the resistant colony. The resistant strain exhibited a 2 -fold lower net reproductive rate $(R o)$, which corresponds to the number of females generated from each original female compared to the susceptible strain. Likewise, the intrinsic growth rate was lower in the resistant strain than that observed in the susceptible and Kisumu strain. The loss in reproductive potential of resistant mosquitoes could be partly attributed to the high levels of resistance and the presence of the metabolic resistance mediating the process ${ }^{17}$. The findings on the reduced reproductive fitness as a result of insecticide resistance agree with other studies ${ }^{16,28}$ on An. funestus and Ae. Aegypti resistant to pyrethroids. In nature the reduced reproductive fitness in mosquitoes carrying resistant genes could result in a decrease in their progeny limiting malaria transmission.

\section{Conclusions}

The study findings shows high fitness cost on the reproductive parameters of pyrethroid resistant mosquitoes particularly on the duration of gonotrophic cycle, fecundity, net reproductive rate and growth intrinsic rates. However, the increased longevity observed in resistant mosquitoes represents a serious threat for disease control, as increased longevity of pyrethroid resistant mosquitoes could lead to an increased level of malaria transmission in regions with high insecticide resistance. The fitness costs observed are likely associated with maintaining both target site and metabolic mechanisms of resistance to pyrethroids. This is critical to determining the extent to which insecticide resistance interacts with mosquito reproduction potential.

\section{Declarations}

\section{Authors' contribution}

JO, MM, EO and YAA conceived and designed the experiments, JO and MM participated in data collection, data analysis and drafted the manuscript. EO, CW, EO, AK, and YAA supervised data collection and contributed to manuscript writing. All authors have read and approved the final manuscript.

\section{Acknowledgments}

The authors acknowledge the Entomology Laboratory at the Kenya Medical Research Institute, Kisumu for providing technical and laboratory space for the study. The permission to publish this study was granted by the director of Kenya Medical Research Institute.

\section{Competing interest}

The authors declare that they have no competing interests. 


\section{Availability of data and materials}

The dataset supporting the conclusions of this article is included within the article

\section{Funding}

This study was supported by grants from the National Institute of Health (R01 A1123074, U19 Al129326, R01 Al050243, D43 TW001505)

\section{References}

1. WHO. World malaria report 2020: 20 years of global progress and challenges. (2020).

2. Ranson, H. et al. Pyrethroid resistance in African anopheline mosquitoes: what are the implications for malaria control? Trends Parasitol, 27, https://doi.org/10.1016/j.pt.2010.08.004 (2011).

3. Hemingway, J. \& Ranson, H. Insecticide Resistance in Insect Vectors of Human Disease. Annuals Review Entomology, 45, 371-391 (2000).

4. Alout, H., Roche, B., Dabiré, R. K. \& Cohuet, A. Consequences of insecticide resistance on malaria transmission. PLoS Pathog, 13, e1006499 (2017).

5. Afrane, Y. A., Zhou, G., Lawson, B. W., Githeko, A. K. \& Yan, G. Effects of Microclimatic Changes Due to Deforestation on the Survivorship and Reproductive Fitness of Anopheles gambiae in Western Kenya Highlands. Am. J., Trop Med Hyg, 74, 772-778 (2006).

6. Lardeux, F. J., Tejerina, R. H., Quispe, V. \& Chavez, T. K. A physiological time analysis of the duration of the gonotrophic cycle of Anopheles pseudopunctipennis and its implications for malaria transmission in Bolivia. Malar. J, 7, 1-17 (2008).

7. Afrane, Y. A., Little, T. J., Lawson, B. W., Githeko, A. K. \& Yan, G. Deforestation Increases the Vectorial Capacity of Anopheles gambiae Giles to Transmit Malaria in the Western Kenya Highlands. Emerg Infect Dis, 10, 1533-1538 (2008).

8. Alout, H., Roche, B., Dabiré, R. K. \& Cohuet, A. J. P. p. Consequences of insecticide resistance on malaria transmission, 13, e1006499 (2017).

9. Vézilier, J., Nicot, A., Gandon, S. \& Rivero, A. J. P. o. t. R. S. B. B. S. Plasmodium infection decreases fecundity and increases survival of mosquitoes. 279, 4033-4041(2012).

10. McCarroll, L. \& Hemingway, J. J. I. b. \& biology, m. Can insecticide resistance status affect parasite transmission in mosquitoes?, 32, 1345 (2002).

11. Platt, N. et al. Target-site resistance mutations (kdr and RDL), but not metabolic resistance, negatively impact male mating competiveness in the malaria vector Anopheles gambiae. 115,243252(2015).

12. Nouage, L. et al. Influence of GST-and P450-based metabolic resistance to pyrethroids on blood feeding in the major African malaria vector Anopheles funestus. (2020). 
13. Rigby, L. M. et al. Identifying the fitness costs of a pyrethroid-resistant genotype in the major arboviral vector Aedes aegypti. 13,1-12(2020).

14. Tchouakui, M. et al. Fitness costs of the glutathione S-transferase epsilon 2 (L119F-GSTe2) mediated metabolic resistance to insecticides in the major African malaria vector Anopheles funestus. 9,645(2018).

15. Kumar, S. et al. Diminished reproductive fitness associated with the deltamethrin resistance in an Indian strain of dengue vector mosquito. Aedes aegypti L, 26, 55-64 (2009).

16. Nkahe, D. L. et al. Fitness cost of insecticide resistance on the life-traits of a Anopheles coluzzii population from the city of Yaoundé. Cameroon, 5, 171 https://doi.org/10.12688/wellcomeopenres.16039.2 (2020).

17. Machani, M. G. et al. Phenotypic, genotypic and biochemical changes during pyrethroid resistance selection in Anopheles gambiae mosquitoes. Sci. Rep, 10, 19063 https://doi.org/10.1038/s41598020-75865-1 (2020).

18. Shute, G. T. A Method of Maintaining Colonies of East African Strains of Anopheles Gambiae. Ann. Trop. Med. Parasitol, 50, 92-94 https://doi.org/10.1080/00034983.1956.11685743 (1956).

19. Afrane, Y. A., Zhou, G., Lawson, B. W., Githeko, A. K. \& Yan, G. Life-table analysis of Anopheles arabiensis in western Kenya highlands: effects of land covers on larval and adult survivorship. The American journal of tropical medicine and hygiene, 77, 660-666 (2007).

20. Minakawa, N. et al. Malaria vector productivity in relation to the highland environment in Kenya. 75,448-453(2006).

21. Ranson, H. \& Lissenden, N. Insecticide resistance in African Anopheles mosquitoes: A worsening situation that needs urgent action to mantain malaria control. Parasites Vectors, 32, 187-196 (2016).

22. Klowden, M. J. \& Briegel, H. Mosquito gonotrophic cycle and multiple feeding potential: contrasts between Anopheles and Aedes (Diptera: Culicidae). J. Med. Entomol, 31, 618-622 (1994).

23. Mebrahtu, Y. B., Norem, J. \& Taylor, M. Inheritance of larval resistance to permethrin in Aedes aegypti and association with sex ratio distortion and life history variation. The American journal of tropical medicine and hygiene, 56, 456-465 (1997).

24. Martins, A. J., Bellinato, D. F., Peixoto, A. A., Valle, D. \& Lima, J. B. P. Effect of insecticide resistance on development, longevity and reproduction of field or laboratory selected Aedes aegypti populations. PLoS One, 7, e31889 (2012).

25. Sy, F. A., Faye, O., Diallo, M. \& Dia, I. Effects of insecticide resistance on the reproductive potential of two sub-strains of the malaria vector Anopheles coluzzii. J. Vector Borne Dis, 56, 207-211 https://doi.org/10.4103/0972-9062.289401 (2019).

26. Osoro, J. K. et al. Insecticide resistance exerts significant fitness costs in immature stages of Anopheles gambiae in western Kenya. Malar. J, 20, 1-7 (2021).

27. Telang, A., Frame, L. \& Brown, M. R. Larval feeding duration affects ecdysteroid levels and nutritional reserves regulating pupal commitment in the yellow fever mosquito Aedes aegypti (Diptera: 
Culicidae). J. Exp. Biol, 210, 854-864 https://doi.org/10.1242/jeb.02715 (2007).

28. Brito, L. P. et al. Assessing the effects of Aedes aegypti kdr mutations on pyrethroid resistance and its fitness cost. PLoS One, 8, e60878 (2013).

\section{Tables}

\section{Table 1}

Mean duration of gonotrophic cyle and fecundity of resistant and susceptible colonies of An.gambiae

Colony/strain

No. laid

Cycle length (days) *

Fecundity* mean \pm SD

mean \pm SD

\begin{tabular}{lcccc}
\hline Resistant & 300 & 94 & $6.1 \pm 0.0 .8^{\mathrm{a}}$ & $88.02 \pm 20^{\mathrm{a}}$ \\
\hline Susceptible & 300 & 161 & $4.3 \pm 1.1^{\mathrm{b}}$ & $104 \pm 28^{\mathrm{b}}$ \\
\hline Kisumu & 300 & 197 & $3.7 \pm 0.2^{\mathrm{b}}$ & $97 \pm 34^{\mathrm{a}}$
\end{tabular}

*Values representing the percentage of mosquitoes that laid eggs per colony, mean and standard deviations of gonotrophic cycle length in days and fecundity. The same superscript letters indicate no significant difference.

Gonotrophic cycle is the number of days from blood feeding to egg laying.

Fecundity is measured in number of eggs laid per female.

\section{Table 2}

Comparison of adult longevity, fecundity, net reproductive rate and intrinsic growth rate among the resistant, susceptible and Kisumu colonies 


\section{Mean \pm SD}

\begin{tabular}{lllll} 
Colony/Strain & longivity (days) & $\mathbf{R}_{\mathbf{0}}$ & $\boldsymbol{r}$ & Fecundity \\
\hline Resistant & $39.7 \pm 1.6^{\mathrm{a}}$ & $2.6 \pm 0.3^{\mathrm{a}}$ & $0.215 \pm 0.007^{\mathrm{a}}$ & $4.31 \pm 0.9^{\mathrm{a}}$ \\
\hline Susceptible & $29.9 \pm 1.7^{\mathrm{b}}$ & $4.7 \pm 0.6^{\mathrm{b}}$ & $0.285 \pm 0.010^{\mathrm{b}}$ & $8.6 \pm 1.0^{\mathrm{b}}$ \\
\hline Kisumu strain & $29.6 \pm 1.1^{\mathrm{b}}$ & $4.1 \pm 0.2^{\mathrm{b}}$ & $0.241 \pm 0.013^{\mathrm{b}}$ & $9.7 \pm 0.1^{\mathrm{b}}$
\end{tabular}

*Mean and standard deviations of fecundity, net reproductive rate intrinsic per- capita growth rate and survival time. The same superscript letters indicate no significant difference.

Fecundity is measured in number of female progeny per female per day.

$R 0$ - is the mean net replacement rate (number of offspring per female per generation).

$r$-is intrinsic per-capita growth rate (number of offspring per female per day).

\section{Figures}




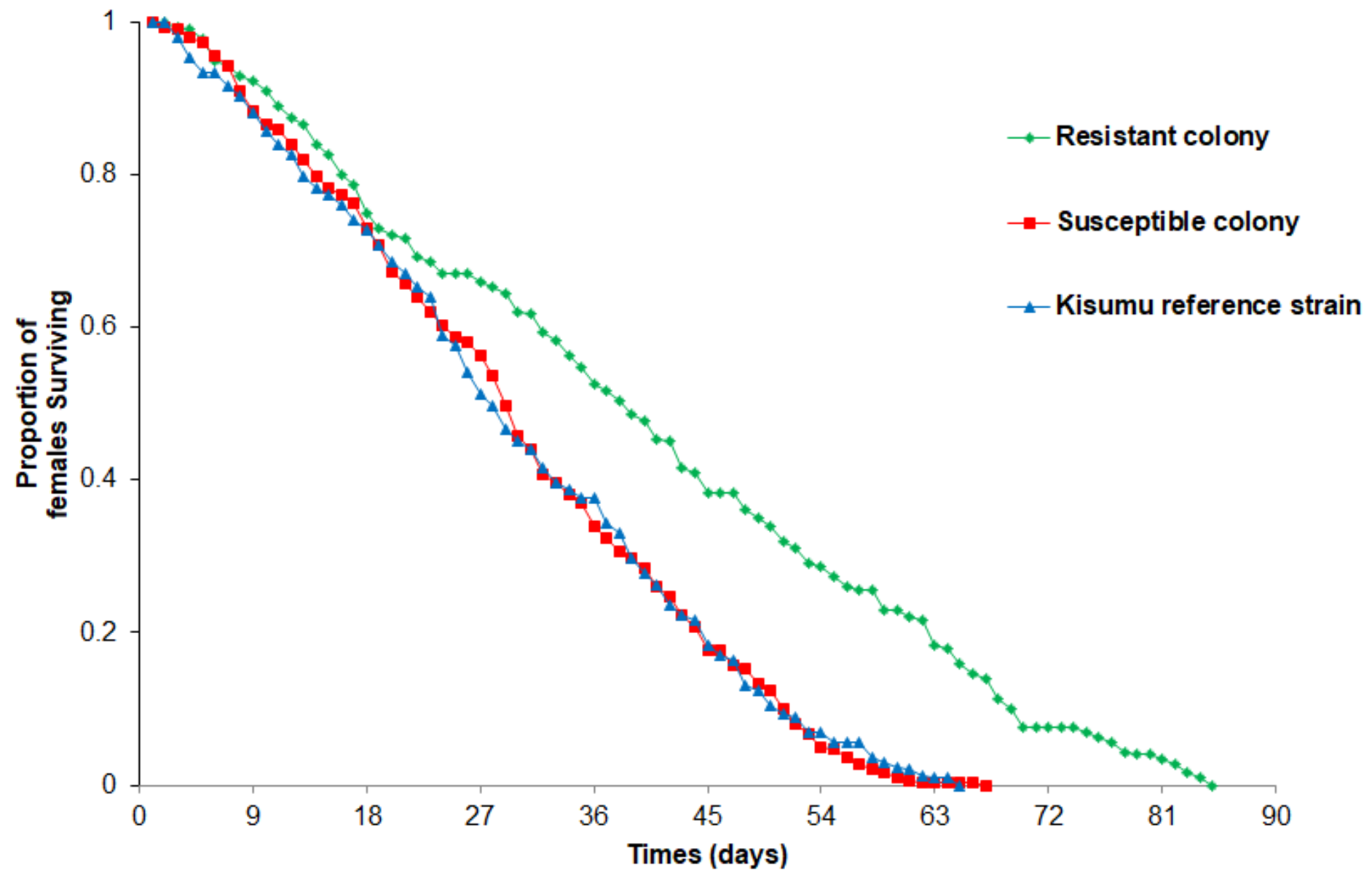

Figure 1

Comparison of survivorship among the resistant, susceptible and Kisumu strain An. gambiae females 